\title{
Design of Multi Granularity Quantum Switching Node Structure for Embedded Intelligent System
}

\author{
Liu Xingjian ${ }^{\mathrm{a}, *}$, Chen Xiao \\ School of Computing, Guangdong Business and Technology University, Zhaoqing, Guangdong, 526060, \\ China \\ a email: starsword2@163.com \\ *corresponding author
}

Keywords: Power Man, Embedded Intelligent System, Multi Granularity, Quantum Switching

\begin{abstract}
The load-bearing modes of power man services are quite different. Network switching nodes not only need to support the exchange of quantum signal, synchronous signal and classic signal, but also need to have multi granularity signal exchange function to support the encrypted transmission of power services with different service levels. The multi granularity quantum switch node structure based on fiber level switch layer, band level switch layer and wavelength level switch layer is adopted to improve the flexibility of switch node and reduce the scale of cross connection matrix. At the same time, the wavelength selection switch is introduced into the multi granularity quantum switching node, which ensures the same path exchange between the quantum signal and the synchronous signal of the same user through the programmable flexible wavelength selection. The simulation results show that the quantum switch node structure is feasible in the application of power man.
\end{abstract}

\section{Introduction}

Security problem is the key problem that must be solved in the way of information and intelligence in power industry. Quantum key distribution can be an important technology to ensure the security of power communication network[1]. For power wide area network, node exchange is the main technology to expand the network scale. The power man is an important part of the power Wan. It can realize the hybrid transmission of many different bearer network services, such as power control special line business, protection security $2 \mathrm{~m}$ channel business, management information business, video business and voice exchange business[2]. The communication modes of these power special services are different, some are directly transmitted through optical fiber, some are transmitted through special channel, some are transmitted through IP network, and the bearer network is also different, which is completely isolated physically. Because the introduction of QKD will increase the number of wavelength channels, which will greatly increase the scale of cross connection matrix and lead to the increase of insertion loss, it is necessary to control the insertion loss of switching nodes to ensure the performance of QKD[3]; on the other hand, for the QKD synchronous signal, the same user's quantum signal and synchronous signal need to maintain the consistency of transmission path to achieve the normal detection of the receiver, it is necessary to The switching node has flexible wavelength channel selection function. Therefore, the core nodes of the network need to have the processing capabilities of different optical fibers, different wavelengths and different granularity services, and support the exchange of quantum signals, synchronous signals and classic optical signals. In order to solve these two problems, this paper introduces a multi granularity quantum switching node structure for power man.

\section{Research Status of Quantum Switching Node}

At present, the academic research on QKD network is in full swing, but its scale is generally small. China University of science and Technology (USTC) proposes a full time all pass quantum 
circuit based on WDM. The quantum router consists of four WDM devices, WDM a, WDM B, WDM C and WDM D. each WDM device has three wavelengths, and the multiplexing / demultiplexing wavelengths supported are $\lambda_{1}, \lambda_{2}$ and $\lambda_{3}$. WDM A and WDM D exchange signals through wavelength $\lambda 2$, WDM A and WDM B exchange signals through wavelength $\lambda_{3}$, WDM A and WDM $C$ exchange signals through wavelength $\lambda_{1}$, and the connections between other WDM devices are the same, so complete internal connections can be completed accordingly[4]. Based on this kind of quantum router, a full-time and all pass QKD network can be established, which can complete the QKD between different nodes.

A quantum switching node based on optical add drop multiplexer is proposed by the University of technology in Madrid. The signal enters the node through the input port, and outputs the wave length matched signal from the filter port through the band-pass filter FQ, and the unmatched signal from the reflection port. The principle of FC is the same as that of FQ, which outputs the wavelength matched classical signal from the filter port by setting the center wavelength. The classical signal and quantum signal filtered out at the filter port will be multiplexed with WDM equipment through the circulator and transmitted to the access network. When the signals transmitted from the access network enter the node, the WDM device will separate the quantum signals from the classical signals[5]. These signals bypass the filter through the circulator, are reused in another WDM device, and then arrive at the destination through the ring backbone network. The node can complete the automatic addressing function, and the passive optical devices used are relatively simple and easy to implement.

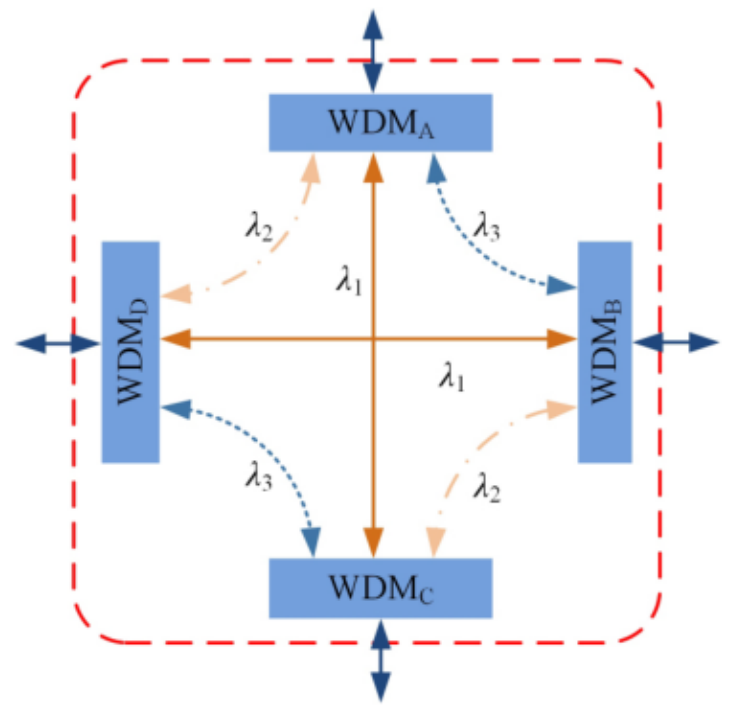

Figure 1 Four port all time all pass quantum router

From the above analysis, it can be seen that the node structure proposed by the University of science and technology of China can not realize dynamic configuration, and there are many limitations in practical applications, such as wavelength cannot be changed, time-sharing cannot be used, etc. In addition, although the quantum switching node based on OADM proposed by Madrid University of technology can realize automatic addressing function, it can only support single fiber link transmission, and has low flexibility. Therefore, we need to propose a new switching node structure, which not only has the characteristics of dynamic configuration, but also can realize the same node switching of classical, synchronous and quantum signals.

\section{Three Layer Architecture of Multi Granularity Quantum Switching Node}

As mentioned before, in the electric power communication network, due to the different loadbearing modes and communication modes of various electric power services, the service quality varies greatly[6]. The most obvious is that the production control service and management 
information service are two independent load-bearing networks, which are physically isolated and have different requirements on time delay, reliability and protection. Therefore, for the core communication nodes, to meet the flexible transmission of multiple types of services, when the local services are uploaded to the far end or the services are downlink from the convergence layer to the local, it is necessary to transfer the signal from the fiber granularity to the wavelength granularity and then continue the transmission from the switching node. In addition, when the convergence layer converges different services for transmission, although there is no need to switch at a smaller granularity level in the switching node, it still needs to switch at a larger granularity level (such as waveband, optical fiber). In order to realize the integration of QKD network and power man, the exchange nodes in the network not only need to support the same node exchange of quantum signals, synchronous signals and classic signals, but also need to have the ability of flexible exchange of multi granularity signals to ensure the information security transmission of power information network. Therefore, this paper proposes a multi granularity quantum switch node structure based on fiber level switch layer, band level switch layer and wavelength level switch layer. The node structure is composed of FSL, BSL and WSL[7]. FSL is a simple structure composed of an optical fiber cross connect module, and the core of FXC module is an optical switch matrix of $\mathrm{M} \times \mathrm{m}[8]$; BSL is composed of a wavelength selective switch device and a band cross connect module, while the core of bxc module is an optical switch matrix of $n \times n$; WSL is composed of a dense optical wavelength multiplexing device and a wavelength cross connect module The core of WXC module is $\mathrm{k} \times \mathrm{K}$ optical switch matrix. Suppose that there are $\mathrm{x}$ optical fiber links from adjacent switching nodes to FSL of this switching node, which is the proportion of signals in FSL to be demultiplexed to BSL, and $\mathrm{m} \times \mathrm{x}$ is the number of ports from FSL to BSL. FSL signal is demultiplexed as the input of BSL[9]. The number of input wave bands is $y, n$ is the proportion of signal demultiplexed to WSL in BSL, and $\mathrm{N} \times \mathrm{y}$ is the number of ports from BSL to WSL. Due to the symmetry of this structure, the number of ports from FSL to BSL is the same as that from BSL to FSL, and the number of ports from WSL to BSL is the same as that from BSL to WSL. By introducing WSS device into BSL, the node realizes the path unification of quantum and synchronous signals of the same user in the exchange process. WSS devices not only have dynamic configuration, but also can exchange different wavelengths flexibly. By introducing WSS devices into the switching structure, the switching capability is greatly enhanced, and its construction cost and difficulty are further reduced.

\section{Structure and Performance Analysis of Multi Granularity Quantum Switching Nodes}

A simple typical power man topology composed of multi granularity quantum switching nodes. Each switching node is connected with several adjacent switching nodes respectively. Classic power services and QKD services of different granularity are switched to corresponding output ports through their respective switching levels, so as to realize flexible routing function. At the same time, each level of each switching node has uplink and downlink interfaces to connect with the corresponding access network services, so as to realize the uplink and downlink functions of power services in different bearing modes. Suppose that a classical power service in access network $\mathrm{B} 1$ needs to go through node a to access network D1; the destination of quantum signal is access network A2; access network B2 is dedicated power line service (such as security control service) and the destination is access network C1. Two signals of syn and QKD are directly exchanged at node $\mathrm{B}$ to the output port connected with node a through fiber level exchange (Note: the direct dotted line between a and B is a QKD logical link); in node a, the quantum signal is required to be routed down, and the synchronous signal of the user is guaranteed to be exchanged along the same path with the quantum signal. This function is realized by the bxc layer of the switching node through configuration WSS of bxc layer realizes that quantum signal and synchronous signal are always output from the same output port, and are downed from the port connected with access network A2; at the same time, classic power business is switched to node $\mathrm{D}$, and is directly output to access network D1 through the down port of optical fiber level; the latter B2 special line signal is directly switched to access network C1 through optical fiber. In this way, the flexible exchange and 
transmission of power business with different quality levels and multiple bearing modes are realized. Compared with the optical switching node structure proposed by the single granularity switching node structure, the multi granularity switching node structure not only overcomes the problems of the former two, but also can use a variety of granularity to carry services. Moreover, by introducing WSS device into BSL, it ensures the same path transmission of quantum signal and synchronous signal, improves the performance of the switching node, and reduces its complexity. In addition, multi granularity switching nodes can also undertake the switching transmission of larger services without increasing the number of ports of multiplexing / demultiplexer and optical switch matrix, so to some extent, it can improve the service capacity of switching nodes.

The forwarding delay of multi granularity switching node mainly depends on the switching delay of cross connection matrix and the switching delay of wavelength selective switch. MEMS optical switch based on MEMS has the advantages of low cost, easy integration and millisecond switching speed. Its delay meets the technical requirements of DWDM all-optical network. Therefore, MEMS optical switch becomes the main switch of multi granularity switching node cross connection matrix. Now, the switching time of commercial wavelength selective switch is more than milliseconds, which can meet the requirements of high-speed optical communication system. Therefore, the multi granularity switching node structure can support the current lower rate quantum communication on the basis of the existing commercial devices and can meet the needs of higher rate optical communication at the same time. For the power man, because the power network is mostly static configuration service, it is not sensitive to the switching delay of the service, and the switching time of the optical switch does not affect the reliability of the power communication network.

\section{Conclusion}

FSL, BSL, WSL are based on the multi granularity quantum switch node structure. The same node of quantum signal, synchronous signal and classical power signal not only realizes the exchange, but also encrypts the transmission of power services by various azimuth methods. In order to support the multi granularity signal, they have the exchange function. Based on the analysis of multi granularity quantum switching node's exertion, reversibility and distortion, the feasibility of proving the applicability of the multi-level quantum switching node structure of power communication man is constructed.

\section{References}

[1] Frederick C. I Stochastic Granular Space-time and Quantum Mechanics, 2017.

[2] Daniel Nagy, Guillermo Indalecio, Antonio J. Garcia-Loureiro,. I Metal Grain Granularity Study on a Gate-All-Around Nanowire FET. IEEE Transactions on Electron Devices, no. 99, pp. 1-7, 2017.

[3] Xiuli Wu, Shaomin Wu. I An elitist quantum-inspired evolutionary algorithm for the flexible job-shop scheduling problem. Journal of Intelligent Manufacturing, vol. 28, no. 6, pp. 1441-1457, 2017.

[4] Alexey A. Melnikov, Hendrik Poulsen Nautrup, Mario Krenn,. I Active learning machine learns to create new quantum experiments. Proceedings of the National Academy of Sciences, vol. 115, no. 6, pp. 1221-1226, 2017.

[5] Roman A. Perez, Rajendra K. Singh, Tae-Hyun Kim,. I Silica-based multifunctional nanodelivery systems toward regenerative medicine. Materials Horizons, no. 4, 2017.

[6] Chang-Hong Hsu, Yunqi Zhang, Michael A. Laurenzano,. I Reining in Long Tails in Warehouse-Scale Computers with Quick Voltage Boosting Using Adrenaline. Acm Transactions on Computer Systems, vol. 35, no. 1, pp. 1-33, 2017.

[7] Yuanzhen Li, Yang Zhao, Yingyu Zhang. I A spanning tree construction algorithm for industrial 
wireless sensor networks based on quantum artificial bee colony. EURASIP Journal on Wireless Communications and Networking, vol. 2019, no. 1, 2019.

[8] H. Hu, K. Yang, Y. Liang. I Economic operation for hydropower station using cloud adaptive quantum-inspired binary social spider optimization. Journal of Huazhong University of Science \& Technology, vol. 46, no. 3, pp. 122-127, 2018.

[9] Oleg E, Ibragimovna A G. ICT through the prism of critical technologies, vol. 19, no. 2, 2017. 